Cultures \& Conflits

06 | été 1992

Violences urbaines

\title{
Editorial. Approche comparée des violences urbaines
}

\section{Michel Wieviorka}

\section{(2) OpenEdition \\ 1 Journals}

\section{Édition électronique}

URL : http://journals.openedition.org/conflits/649

DOI : 10.4000/conflits.649

ISSN : $1777-5345$

Éditeur :

CCLS - Centre d'études sur les conflits lilberté et sécurité, L'Harmattan

\section{Édition imprimée}

Date de publication : 16 juillet 1992

ISSN : 1157-996X

\section{Référence électronique}

Michel Wieviorka, « Editorial. Approche comparée des violences urbaines », Cultures \& Conflits [En ligne], 06 | été 1992, mis en ligne le 07 janvier 2003, consulté le 30 mars 2021. URL : http:// journals.openedition.org/conflits/649; DOI : https://doi.org/10.4000/conflits.649

Ce document a été généré automatiquement le 30 mars 2021.

Creative Commons License 


\title{
Editorial. Approche comparée des violences urbaines
}

\author{
Michel Wieviorka
}

1 Les violences urbaines contemporaines traduisent-elles la décomposition des nations, la crise ou la mutation des sociétés où elles surgissent, l'affaiblissement des Etats? Lorsqu'elles sont l'expression d'une révolte qui constitue une demande d'intégration bien plus qu'une volonté de rupture, ne posent-elles pas des problèmes éthiques et moraux à ceux qui exercent la responsabilité du pouvoir, aux acteurs politiques, aux intellectuels? Au nom de quelles valeurs les gouvernants peuvent-ils maintenir un ordre acceptable face aux violences des exclus? Le comportement des forces de l'ordre ne renforce-t-il pas trop souvent l'exclusion et la spirale de la violence? Telles sont quelques unes des questions, parmi d'autres, que posent les auteurs qui contribuent au présent numéro de notre revue.

2 Les violences urbaines, aujourd'hui, ne semblent guère s'inscrire dans une continuité historique, qui les apparenterait aux poussées révolutionnaires ou aux grèves insurrectionnelles du passé, elles ne semblent pas porter le rêve ou les utopies d'un nouveau modèle de société, même si elles en appellent parfois à l'accouchement d'un monde plus juste. Destructrices, elles comportent généralement une charge d'autodestruction dans laquelle s'abolissent des individus exclus ou marginalisés par rapport à l'emploi, mais aussi à la consommation telle que les couches moyennes la donnent à voir, l'orientent et la pratiquent. Et face aux éruptions et aux débordements de violence, le discours moral de ces mêmes couches moyennes, largement repris par les médias européens ou américains, non seulement constitue une réponse inappropriée, mais crée aussi des effets de stigmatisation des individus et des groupes, et de négation de leurs problèmes sociaux qui ne peuvent que renforcer la radicalisation de leurs conduites. Le contraste est saisissant, pour certains Etats, entre leur capacité d'action dans l'ordre international, qui demeure considérable et leur paralysie ou leur impuissance face aux problèmes de société. L'effondrement du communisme a privé l'ordre international d'un principe central de structuration et d'opposition. N'a-t-il pas aussi affecté l'ordre interne, affaiblissant, par exemple, les 
mécanismes d'intégration qui avaient relativement bien fonctionné en Europe occidentale, pour les Italiens du Mezzogiorno venus travailler à Milan ou pour les Polonais émigrés en Lorraine? Face aux incertitudes, à l'insécurité, la tentation des Etats n'est-elle pas de monter contre les " sauvages urbains ", ces nouvelles classes dangereuses, des opérations de type " vigipirate ", d'adopter une attitude purement répressive? Démarche dangereuse, et dont les résultats peuvent vite s'avérer producteurs de ce qu'il s'agit d'éradiquer, en enfermant des groupes entiers dans une définition ethnique, voire radicale, autant imposée du dehors que voulue ou assumée, en enclenchant ou en accélérant une dialectique des identités, qui fait qu'à toute affirmation identitaire d'un groupe ne peut que correspondre, en réponse l'affirmation d'autres groupes, et en interdisant aux individus qui refusent de se reconnaître dans les solidarités primordiales d'un groupe d'appartenance prescrite d'échapper à ces perceptions extérieures sommaires.

3 Dans les perceptions stigmatisantes, comme dans les solidarités inventées, l'interdépendance de l'interne et de l'externe ne saurait être négligée. Ainsi, la guerre du Golfe a fait craindre, en France, pour la paix dans les banlieues, elle a suscité le recours par l'État à des intermédiaires communautaires, mais l'a aussi encouragé à imposer des contraintes renforçant le processus de stigmatisation des immigrés. Symétriquement, les références positives à Saddam Hussein, à l'Intifada ou, plus récemment au fis, servent parfois aux jeunes issus de l'immigration pour exprimer leurs demandes sociales, mais aussi leur rage et une frustration qui deviennent durables.

4 Mais que peut faire un Etat dans de telles conditions? Ne voit-on pas de toutes parts monter une critique qui condamne les pratiques administratives et dénonce les promesses des " politiciens voleurs ", les uns passant à la violence émeutière, mais aussi au repli sur soi, à la drogue, ou à la fermeture identitaire, les autres, selon une logique de " pauvres Blancs ", versant dans un populisme qui traduit un fort sentiment d'abandon, un rejet croissant de la modernité, une peur pour l'identité nationale ou religieuse. D'un côté la violence, l'exclusion de minorités à qui les réseaux de la mobilité sociale semblent interdits, de l'autre l'appel à des solutions politiques extrêmes, ou à la privatisation d'un ordre que l'Etat serait incapable d'assurer : l'Etat semble impuissant, tandis que l'hypothèse de l'accroissement des violences, individuelles et collectives, est à l'ordre du jour. Toute une évolution est ainsi mise en cause, où la mise en scène de la violence avait progressivement, croyait-on, remplacé sa présence dans la vie collective. Les intermédiaires du passé, partis de masse ou syndicats, ont perdu leur capacité à assurer la représentation des demandes sociales, et les groupes que l'on retrouve le plus souvent dans les violences urbaines se prêtent mal, ne serait-ce que par leur hétérogénéité et la radicalisation de leur action, à la construction de mécanismes d'intégration conflictuelle. Tendus vers la rupture, ils résistent à l'intervention de médiateurs qui permettraient de rétablir le contact, et en qui ils voient vite soit des représentants du système qui les exclut, soit des notables dont ils se dissocient.

5 Les références identitaires, ethniques et religieuses notamment, sont-elles susceptibles d'animer une action qui ne soit pas de rupture ou d'auto-enfermement, et qui soit inscrite dans un système ouvert, démocratique, orienté par des valeurs universelles suffisamment souples pour que ne soit pas exigé l'écrasement des particularismes? Il serait illusoire de s'en remettre à des stratégies répressives, à des logiques policières 
oscillant entre le pur usage de la force, et l'appel à des mécanismes de contrôle communautaire : il n'y a pas de solution technique ou instrumentale à la violence. On en revient au problème éthique et moral des fondements de l'action politique. A quelles conditions, en fonction de quelles orientations générales une société peut-elle faire reculer l'exclusion, réintroduire de la relation là où domine la rupture, susciter les médiations et les formes de représentation dont l'absence encourage la violence?

\section{RÉSUMÉS}

Les violences urbaines contemporaines traduisent-elles la décomposition des nations, la crise ou la mutation des sociétés où elles surgissent, l'affaiblissement des Etats ? Lorsqu'elles sont l'expression d'une révolte qui constitue une demande d'intégration bien plus qu'une volonté de rupture, ne posent-elles pas des problèmes éthiques et moraux à ceux qui exercent la responsabilité du pouvoir, aux acteurs politiques, aux intellectuels? Au nom de quelles valeurs les gouvernants peuvent-ils maintenir un ordre acceptable face aux violences des exclus ? Le comportement des forces de l'ordre ne renforce-t-il pas trop souvent l'exclusion et la spirale de la violence ? Telles sont quelques unes des questions, parmi d'autres, que posent les auteurs qui contribuent au présent numéro de notre revue.

Concerning the prolongation of urban riots, the first requirement is the analysis of urban violence present at large in western societies. What are the reasons for the violence in the cities of France, the United Kingdom or the United States ? Discussion should include politics, transnational relations and the dynamics of violence occurring during confrontations between police forces and so-called ethnic groups. Perhaps a better understanding of the reasons for the emergence of violence may come through " intermediate " theories, that is, less general and more related to the specific context of the particular events, the representations and the values of the actors.

\section{INDEX}

Mots-clés : villes/ sociologie urbaine, violence 